\title{
Radiofrequency Ablation with the Real-Time Virtual Sonography System for Treating Hepatocellular Carcinoma Difficult to Detect by Ultrasonography
}

\author{
Hiroaki Kawasoe ${ }^{1}$, Yuichiro Eguchi ${ }^{1, *}$, Toshihiko Mizuta ${ }^{1}$, Tsutomu Yasutake ${ }^{1}$, Iwata Ozaki ${ }^{1}$, \\ Tomonori Shimonishi' ${ }^{2}$, Kohji Miyazaki ${ }^{2}$, Tsutomu Tamai ${ }^{3}$, Akira Kato ${ }^{3}$, Sho Kudo ${ }^{3}$, and \\ Kazuma Fujimoto ${ }^{1}$ \\ ${ }^{1}$ Department of Internal Medicine, Saga Medical School, 5-1-1, Nabeshima, Saga 849-8501, Japan \\ ${ }^{2}$ Department of Surgery, Saga Medical School, 5-1-1, Nabeshima, Saga 849-8501, Japan \\ ${ }^{3}$ Department of Radiology, Saga Medical School, 5-1-1, Nabeshima, Saga 849-8501, Japan
}

Received 21 August, 2006; Accepted 5 September, 2006

\begin{abstract}
Summary Radiofrequency ablation has been applied to treat hepatocellular carcinoma, with favorable therapeutic outcomes. Nevertheless, practitioners have approached radiofrequency ablation with some reluctance due to the difficulty of identifying isoechoic tumors and recurrent tumors. The aim of the present study is to investigate the efficacy of Real-time Virtual Sonography to treat hepatocellular carcinoma difficult to detect by conventional ultrasonography. Real-time Virtual Sonography is a system generating multiplanar reconstruction images in real-time using the Hitachi medico EUB-8500 equipped with a probe. The system included following components: 1) digital imaging and communications in medicine (DICOM) data from dynamic CT, 2) a magnetic field generator to match the multiplanar reconstruction image on the monitor and the actual ultrasonography image, 3) the cross section with the tumor displayed as a multiplanar reconstruction image. Total twenty-five nodules of twentyone patients underwent radiofrequency ablation monitored by Real-time Virtual Sonography. All nodules difficult to detect via conventional ultrasonography were clearly visualized in realtime. The average nodule diameter was $2.4 \pm 1.6 \mathrm{~cm}$, and punctures and coagulation were performed an average of 2.2 and 3 times per session. Dynamic CT after session confirmed effective coagulation of each nodule. In conclusion, this study demonstrates that the present system is capable of effectively and accurately treating tumors difficult to detect by conventional ultrasonography.
\end{abstract}

Key Words: multiplanar reconstruction image, dynamic CT, hepatic tumor, HCV, coagulation

\section{Introduction}

The incidence of HBV or HCV-associated viral hepatitis is increasing [1], along with the incidence of hepatocellular

\footnotetext{
*To whom correspondence should be addressed.

Tel: +81-952-34-2362 Fax: +81-952-34-2017

E-mail: eguchiyu@med.saga-u.ac.jp
}

carcinoma (HCC). However, advances in diagnostic imaging techniques such as ultrasonography, computed tomography (CT), and magnetic resonance imaging (MRI) now enable early detection and diagnosis of HCC [2]. In recent years, HCC detected by these diagnostic imaging techniques has been treated locally by radiofrequency ablation (RFA) and other techniques, with favorable therapeutic outcomes comparable to hepatectomy [3, 4]. However, abdominal ultrasonography does not always provide precise guidance in 
identifying isoechoic tumors, tumors recurring locally in areas treated with lipiodol following transarterial chemoembolization (TACE), or tumors recurring in areas treated by RFA or percutaneous ethanol injection (PEI) procedures. We have recently utilized the approaches which involved preparing multiplanar reconstruction (MPR) images that matched ultrasonography images of lesions and expected puncture routes on a workstation. MPR images were reconstructed by matching ultrasonography images and CT data (digital imaging and communications in medicine (DICOM) data) obtained by prior CT during arterial portography (CTAP) and CT during hepatic arteriography (CTHA) [5], followed by more accurate coagulation based on scans of target areas while referring to the relative positions of surrounding vessels and organs shown of the images. One problem with this technique is that MPR images are prepared in advance, and critical discrepancies can arise in scans of intercostal spaces due to axial rotation of a probe between the MPR and ultrasound images. Seeking to resolve this problem, we investigated the feasibility of Hitachi Medical Real-time Virtual Sonography (RVS), a system that generates MPR images in real-time, as a probe scans over target areas.

\section{Materials and Methods}

\section{Subjects}

Among consecutive subjects who admitted to Saga Medical School with diagnosed HCC from January 2004 to January 2005, candidates for unresectable invasive therapy were eligible for enrollment after informed consent was obtained. Diagnosis was confirmed by needle biopsy or by plural imaging techniques including ultrasonography, dynamic CT, dynamic MRI, and super paramagnetic iron oxide MRI. Subjects with up to 3 nodules less than $3 \mathrm{~cm}$ in diameter were excluded from this study and received transarterial chemo-embolization. CTAP/CTHA was performed for all enrolled subjects; 1) to confirm HCC which showed low density tumor with CTAP, high density tumor with early phase CTHA, and low density tumor with corona enhancement with delayed phase CTHA, 2) to survey multi-centric occurrence of HCC, and 3) to confirm intrahepatic tumor staging [6].

\section{Therapeutic system and procedures}

The therapeutic system used comprised the Hitachi Medico Digital Ultrasound EUB-8500 and Hitachi Medico Workstation RVS (Hitachi Medico. co. Tokyo, Japan), which generate real-time MPR images. The RVS, a revolutionary real-time MPR imaging machine that supports ultrasonography diagnosis developed by Hitachi Medico, consists of a small magnetic sensor attached to a convex-shaped EUB-8500 probe. This magnetic sensor precisely and consistently captures changes in magnetic fields generated by the generator placed on the left flank of a patient and detects the changes in the location, direction, and rotation of the probe scanning of the patient. The RVS instantaneously processes changes in positional information detected by the magnetic sensor and generates real-time MPR images matching cross-sectional images of the abdomen captured by the probe (Fig. 1). The actual procedure is as follows: 1) CTAP/CTHA was performed, followed by transfer of volume data (DICOM data) to the RVS. CTAP data was used because CTAP detects portal and hepatic veins, which was captured by ultrasonography. The positional relationship of a tumor to these vessels is critical for identifying lesions which ultrasonography cannot detect. 2) A magnetic field generator is placed on the left flank of a patient. 3) The sagittal section of the left hepatic lobe is first captured using a probe equipped with a magnetic sensor. The xiphoid process serves as the initial reference point. When a CT image with the tip of the xiphoid process captured before MPR processing matches the tip of the xiphoid process on the actual abdominal ultrasound image, the RVS begins continuous generation of MPR images in real-time, displaying the images on the workstation monitor. 4) A cross-sectional image showing the tumor is displayed with the MPR image, and both images are corrected based on neighboring portal and hepatic veins; and 5 ) the tumor is then punctured and coagulated under RVSguidance in the conventional technique. The RFA device used in the methods were Cool-tip electrode with a 2- or 3$\mathrm{cm}$ exposed tip and a radiofrequency generator (Radionics, Burlington, MA). The procedures were each performed by one of three experienced RFA physicians (Y.E, T.Y, and T.M).

\section{Efficacy assessment}

Abdominal dynamic CT was performed on all patients approximately 3 days and 1 month after the treatment for evaluation after RFA. Complete response was confirmed by dynamic CT showed widely low density area with sufficient safety margin.

\section{Results}

In this study, 21 patients included seven men and fourteen women were enrolled (Fig. 2). Their mean age was 73 years old (range 64-84 years old). All patients were positive for the hepatitis $\mathrm{C}$ virus antibody. Eighteen patients had Child-Pugh class A cirrhosis and 3 patients had class B cirrhosis (mean Child-Pugh score $5.8 \pm 0.8$ ). Twenty five nodules in 21 patients were diagnosed HCC (23 nodules were diagnosed by CTAP/CTHA, 2 nodules were diagnosed with needle biopsy as well differentiated HCC. RFA was performed using the present system to treat all 25 nodules in 21 patients. Of the 25 nodules, 11 nodules were tumors with unclear margins, as seen in representative ultrasonography images shown in Fig. 3, 5 were tumors arising in the vicinity 


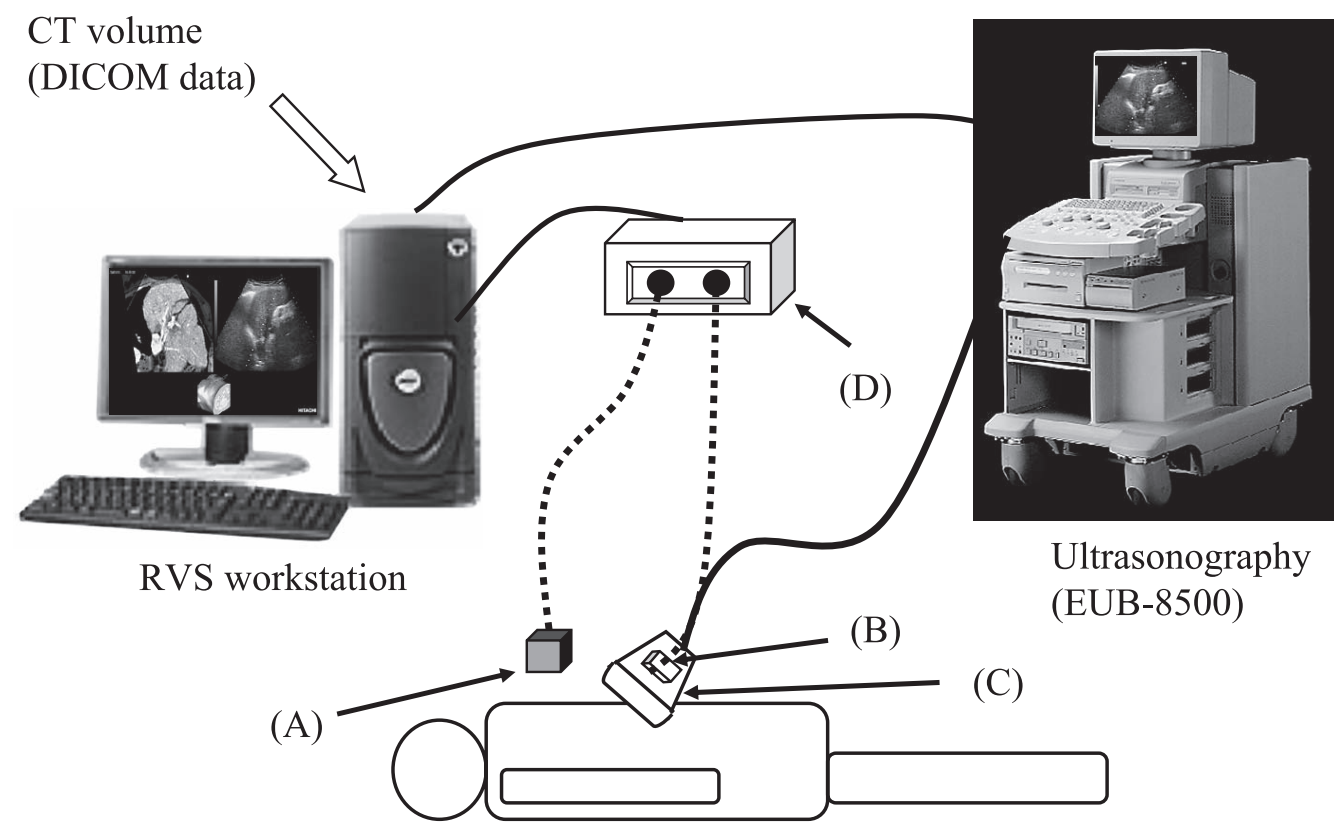

Fig. 1. Summary of the Real-time Virtual Sonography system (Hitachi Medical, partially modified). Step 1: Using the xiphoid process as a reference point, match positional coordinates for positional synchronization. Step 2: Gather positional information from the magnetic sensor attached to the probe. Step 3: An MPR image matching the positional information from the probe is reconstructed based on CT volume data and displayed on the workstation monitor.

A: Magnetic field generator. B: Magnetic sensor. C: Probe. D: Magnetic position detecting unit.

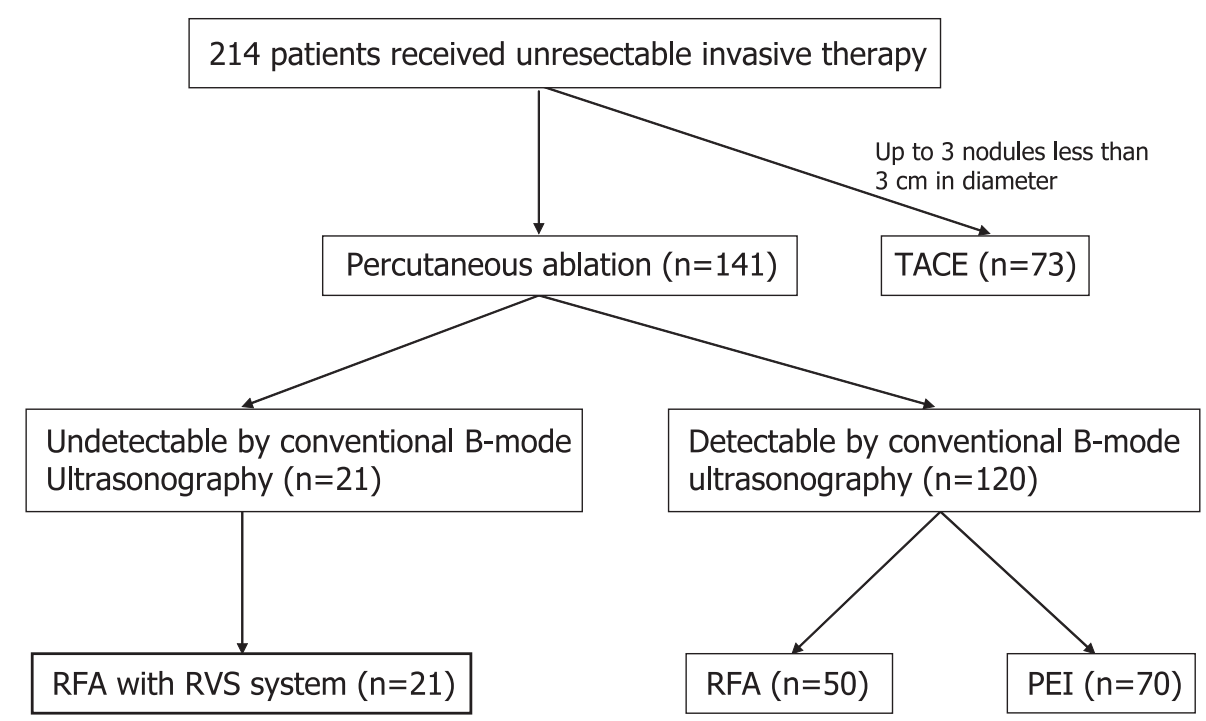

Fig. 2. Flow diagram of subjects enrollment. TACE, transarterial chemo-embolization; RFA, radiofrequency ablation; PEI, percutaneous ethanol injection.

of areas coagulated following radiofrequency ablation; 4 were tumors that could not be fully identified due to their position beneath the diaphragm; 4 were viable tumors emerging following TACE in areas affected by posterior acoustic enhancement of lipiodol deposition; and 1 was a recurrent tumor located at a resection stump following hepatectomy (Table 1).

In all cases, conventional ultrasonography failed to detect the entire lesion accurately. In contrast, the current RVS made it possible to accurately and conveniently ascertain the extent of nodules. RFA was performed using the present system to treat all 25 nodules in 21 patients. The 


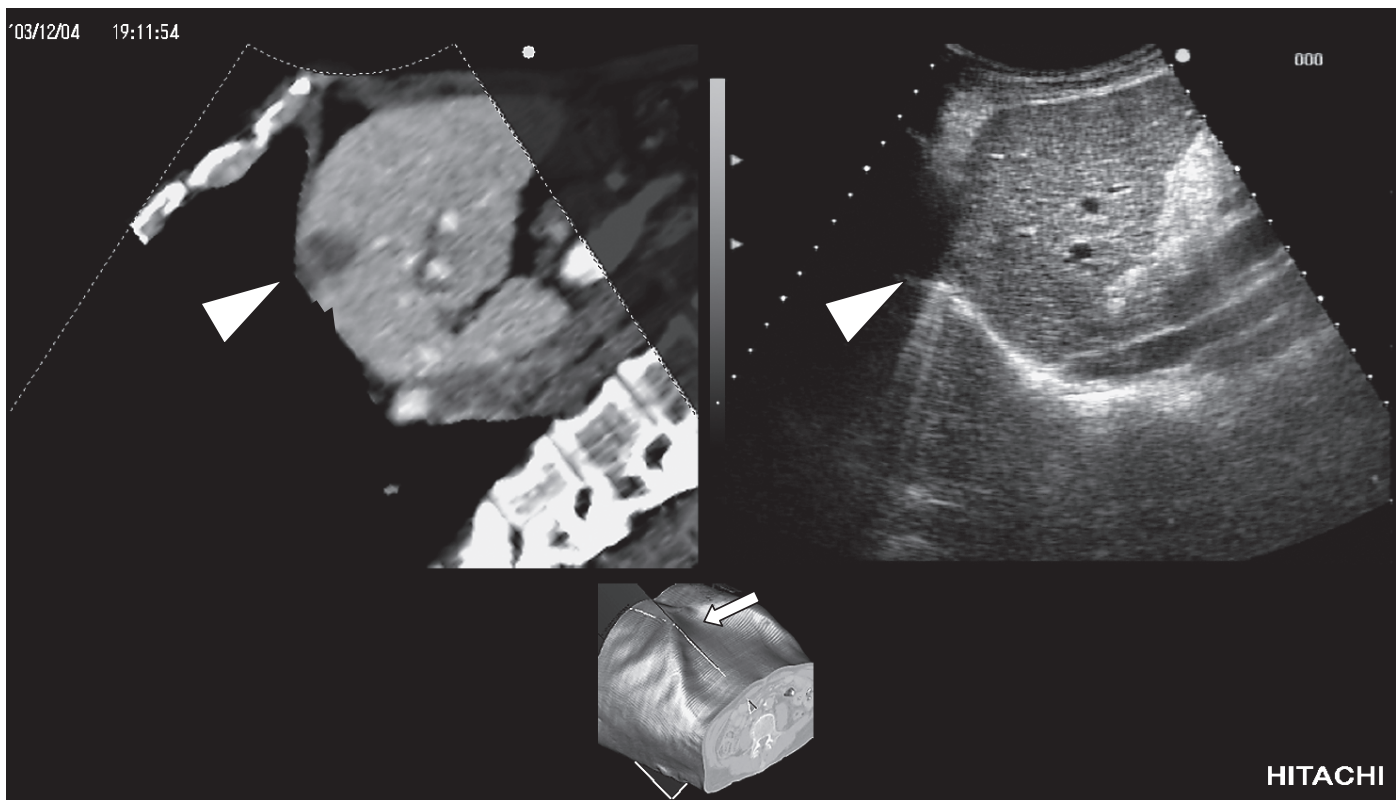

Fig. 3. Images displayed on the workstation monitor. Moving images are displayed in real-time. Upper left: An MPR image reconstructed during scanning. Arrow head: Hepatocellular carcinoma detected by CTAP. Upper right: Actual ultrasound image. The hepatocellular carcinoma was isoechoic and difficult to detect. Lower: A reconstructed image of the trunk region based on CT volume data. The arrow indicates the location of probe scanning ascertained from positional information gathered by the magnetic sensor (arrow). The cross section of this area was reconstructed as an MPR image. MPR: multiplanar reconstruction, CTAP: CT during arterial portography.

Table 1. Characteristics of 25 tumors which were difficult to identify via conventional ultrasonography

\begin{tabular}{|c|c|c|c|c|c|}
\hline & \multirow{2}{*}{ Characteristics of tumors } & \multirow{2}{*}{$\mathrm{n}$} & \multicolumn{3}{|c|}{ Detection (yes/all) } \\
\hline & & & CTAP/CTHA & US & RVS \\
\hline (1) & Unclear margins, as seen in ultrasound images & 11 & $11 / 11$ & $0 / 11$ & $11 / 11$ \\
\hline (2) & $\begin{array}{l}\text { Arising in the vicinity of areas coagulated } \\
\text { following radiofrequency ablation }\end{array}$ & 5 & $5 / 5$ & $0 / 5$ & $5 / 5$ \\
\hline (3) & $\begin{array}{l}\text { Could not be fully identified due to position } \\
\text { beneath the diaphragm }\end{array}$ & 4 & $4 / 4$ & $0 / 4$ & $4 / 4$ \\
\hline (4) & $\begin{array}{l}\text { Viable tumor emerging following transarterial } \\
\text { chemoembolization in areas affected by posterior } \\
\text { acoustic enhancement of lipiodol deposition }\end{array}$ & 4 & $4 / 4$ & $0 / 4$ & $4 / 4$ \\
\hline (5) & $\begin{array}{l}\text { Recurrent tumor located at a resection stump } \\
\text { following hepatectomy }\end{array}$ & 1 & $1 / 1$ & $0 / 1$ & $1 / 1$ \\
\hline
\end{tabular}

average tumor diameter was $2.4 \pm 1.6 \mathrm{~cm}$, and punctures and coagulation were performed an average of 2.2 and 3 times per session. Dynamic CT for evaluation after RFA showed widely low density area with sufficient safety margin and confirmed that each tumor had been effectively coagulated. CTAP image and CTHA images before RFA are shown in Fig. 4. HCC is seen in the area with a reduced portal flow in segment 4 (arrow head) on CTAP (Fig. 4A), the area with high hepatic arterial flow on early-phase CTHA (Fig. 4B), and corona enhancement on delayed-phase CTHA
(Fig. 4C). Fig. 4D shows tumor accurately coagulated under RVS-guidance.

\section{Discussion}

In recent years, $\mathrm{HCC}$ has been treated effectively by combining various therapeutic techniques. Among these techniques, use of percutaneous RFA in particular has grown, with therapeutic outcomes for HCC of less than $3 \mathrm{~cm}$ in diameter comparable to PEI therapy or hepatectomy [3, 

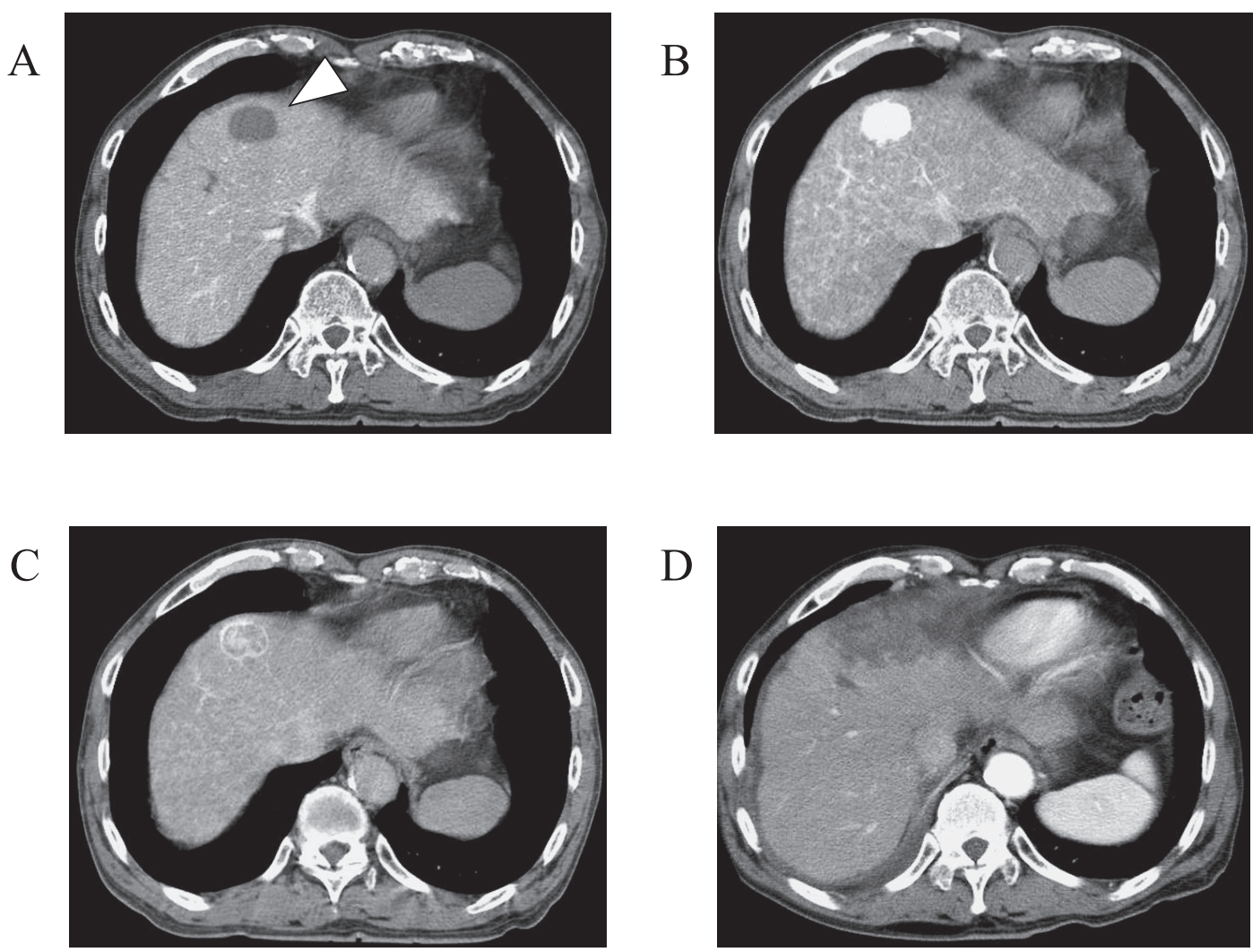

Fig. 4. CT during arterial portography (CTAP) and CT during hepatic arteriography (CTHA) of pre-treatment and dynamic CT of posttreatment of HCC. A: CTAP. Area with a absence of portal flow is shown in segment 4 (arrow head). B: Early-phase CTHA. High hepatic arterial flow and enhanced hepatocellular carcinoma. C: Delayed-phase CTHA. Corona enhancement around the tumor. D: Tumor accurately coagulated under RVS guidance.

7-9]. A recent study shown RFA for HCC is a definitive treatment as a bridge to liver transplantation [10]. But in recent years, treatment has increasingly tackled lesions recurring in the vicinity of areas treated by RFA or TACE [11]. Such recurrent lesions and isoechoic tumors are often difficult to identify by conventional B-mode ultrasonography [12], and imaging success depends heavily on operator's skill [13]. Techniques used to improve the effectiveness of this approach include use of coded-harmonic images and ultrasonographic contrast media; deliberate injection of pleural or peritoneal fluid to aid imaging; and CT-guided punctures [14]. However, each of these methods involves certain drawbacks. We have recently utilized the approaches which involved preparing MPR images that matched ultrasonography images of lesions and expected puncture routes on a workstation. MPR images were reconstructed by prior CT data (DICOM data). Discrepancies can arise between MPR and ultrasound images due to axial rotation of a probe for scans of intercostal spaces. RVS could solve this problem by real-time adjustment based on neighboring portal and hepatic veins, and shapes of the liver bed.

While reports have referenced therapeutic systems that generate MPR images like the RVS items [15] are unable to reconstruct accurate and real-time MPR imaging capable of keeping pace with probe-scanning, the current RVS we employed makes it possible to accurately and conveniently ascertain the extent of nodules which were difficult to identify by conventional ultrasonography. RVS also clearly indicates the location of the lungs, intestines, or mesentery, minimizing the risk of inducing pneumothorax, puncturing the intestinal canal, or burning other organs, even when tumors are located beneath the diaphragm or in the liver bed [16]. These qualities make it an extremely effective approach for treating liver tumors, including cases of HCC, which are not readily identifiable by conventional ultrasonography. Several previous reports indicated that the HCC nodule, which was not detected by conventional B-mode and was detected only by CTAP/CTHA, was detected during surgical operation [17-19]. As indicated in this study, RVS might be one of modalities which recovered defect of conventional B-mode and RVS enabled accurate detection for a target, which supported appropriate therapeutic approach to the HCC nodule.

Some limitations in current RVS systems are as follows. 
First, RVS requires close synchronization of MPR and ultrasound images, making it essential to obtain CT data on portal and hepatic veins, which makes it necessary to investigate RVS systems in patients in whom dynamic CT or dynamic MRI is contraindicated due to issues such as contrast medium allergies or renal dysfunction. Second, the synchronized image on RVS is not perfectly the same slice but the discrepancy in most cases can be adjusted with neighboring portal and hepatic veins, and shapes of the liver bed. In one case in this study, there were no vessels which made reference to detect precise position. Third, it is difficult to lead accurately RFA needles to nodules in some cases, even when RVS can reveal the position of tumor. Because of distortion of the liver, we experienced three nodules those were needed to re-insert for a sufficient puncture. As a result, effective ablations were performed in all cases in this study.

In our current study, the outcome obtained from these patients using RVS systems showed favorable clinical course in spite of these limitations in relatively small sample size. Further study with control group in larger number is required. In conclusion, our study demonstrates that the RVS system is capable of effectively and accurately percutaneous coagulation for HCC which is difficult to detect by conventional ultrasonography.

\section{Acknowledgments}

This study was supported by a Grant-in-Aid from the Ministry of Education, Culture, Sports, Science and Technology, Japan, for Scientific Research, No. 6590606 (TM). The authors would like to thank Naofumi Ono, M.D. (Eguchi Hospital) for excellent advice and Yoichiro Kitajima, R.T. (Eguchi Hospital) for excellent radiological images.

\section{References}

[1] Bruix, J., Sherman, M., Llovet, J.M., Beaugrand, M., Lencioni, R., Burroughs, A.K., Christensen, E., Pagliaro, L., Colombo, M., and Rodes, J.: EASL Panel of Experts on HCC.: Clinical management of hepatocellular carcinoma. Conclusions of the Barcelona-2000 EASL conference. European Association for the Study of the Liver. J. Hepatol., 35, 421-430, 2001.

[2] Bruix, J. and Llovet, J.M.: Prognostic prediction and treatment strategy in hepatocellular carcinoma. Hepatology, 35, 519-524, 2002.

[3] Lam, C.M., Ng, K.K., Poon, R.T., Ai, V., Yuen, J., and Fan, S.T.: Impact of radiofrequency ablation on the management of patients with hepatocellular carcinoma in a specialized centre. Br. J. Surg., 91, 334-338, 2004.

[4] Wakai, T., Shirai, Y., Suda, T., Yokoyama, N., Sakata, J., Cruz, P.V., Kawai, H., Matsuda, Y., Watanabe, M., Aoyagi, Y., and Hatakeyama, K.: Long-term outcomes of hepa- tectomy vs percutaneousablation for treatment of hepatocellular carcinoma $\leq 4 \mathrm{~cm}$. World J. Gastroenterol., 28, 546$552,2006$.

[5] Matsui, O., Takashima, T., Kadoya, M., Ida, M., Suzuki, M., Kitagawa, K., Kamimura, R., Inoue, K., Konishi, H., and Itoh, H.: Dynamic computed tomography during arterial portography: the most sensitive examination for small hepatocellular carcinoma. J. Comput. Assist. Tomogr., 9, 19-24, 1985.

[6] Matsui, O.: Imaging of multistep human hepatocarcinogenesis by CT during intra-arterial contrast injection. Intervirology, 47, 271-276, 2004.

[7] Lencioni, R., Cioni, D., Crocetti, L., Franchini, C., Pina, C.D., Lera, J., and Bartolozzi, C.: Early-stage hepatocellular carcinoma in patients with cirrhosis: long-term results of percutaneous image-guided radiofrequency ablation. Radiology, 234, 961-967, 2005.

[8] Lin, S.M., Lin, C.J., Lin, C.C., Hsu, C.W., and Chen, Y.C.: Radiofrequency ablation improves prognosis compared with ethanol injection for hepatocellular carcinoma $<4 \mathrm{~cm}$. Gastroenterology, 127, 1714-1723, 2004.

[9] Lencioni, R.A., Allgaier, H.P., Cioni, D., Olschewski, M., Deibert, P., Crocetti, L., Frings, H., Laubenberger, J., Zuber, I., Blum, H.E., and Bartolozzi, C.: Small hepatocellular carcinoma in cirrhosis: randomized comparison of radiofrequency thermal ablation versus percutaneous ethanol injection. Radiology, 228, 235-240, 2003.

[10] Lu, D.S., Yu, N.C., Raman, S.S., Lassman, C., Tong, M.J., Britten, C., Durazo, F., Saab, S., Han, S., Finn, R., Hiatt, J.R., and Busuttil, R.W.: Percutaneous radiofrequency ablation of hepatocellular carcinoma as a bridge to liver transplantation. Hepatology, 41, 1130-1137, 2005.

[11] Horiike, N., Iuchi, H., Ninomiya, T., Kawai, K., Kumagi, T., Michitaka, K., Masumoto, T., and Onji, M.: Influencing factors for recurrence of hepatocellular carcinoma treated with radiofrequency ablation. Oncol. Rep., 9, 1059-1062, 2002.

[12] Scaife, C.L. and Curley, S.A.: Complication, local recurrence, and survival rates after radiofrequency ablation for hepatic malignancies. Surg. Oncol. Clin. N. Am., 12, 243-255, 2003.

[13] Poon, R.T., Ng, K.K., Lam, C.M., Ai, V., Yuen, J., Fan, S.T., and Wong, J.: Learning curve for radiofrequency ablation of liver tumors prospective analysis of initial 100 patients in a tertiary institution. Ann. Surg., 239, 441-449, 2004.

[14] Shimizu, M., Iijima, H., Horibe, T., Yamada, M., Suzuki, S., Yanagisawa, K., Seki, T., and Moriyasu, F.: Usefulness of contrast-enhanced ultrasonography with a new contrast mode, Agent Detection Imaging, in evaluating therapeutic response in hepatocellular carcinoma treated with radiofrequency ablation therapy. Hepatol. Res., 29, 235-242, 2004.

[15] Hirooka, M., Iuchi, H., Kurose, K., Kumagi, T., Horiike, N., and Onji, M.: Abdominal virtual ultrasonographic images reconstructed by multi-detector row helical computed tomography. Eur. J. Radiol., 53, 312-317, 2005.

[16] Hori, T., Nagata, K., Hasuike, S., Onaga, M., Motoda, M., Moriuchi, A., Iwakiri, H., Uto, H., Kato, J., Ido, A., Hayashi, 
K., and Tsubouchi, H.: Risk factors for the local recurrence of hepatocellular carcinoma after a single session of percutaneous radiofrequency ablation. J. Gastroenterol., 38, 977-981, 2003.

[17] Fujishima, T., Yoshida, H., Obi, S., Shiina, S., Kanda, M., Tateishi, R., Akamatsu, M., Koike, Y., Sato, S., Teratani, T., Kawabe, T., Shiratori, Y., and Omata, M.: Analysis of factors influencing hepatocellular carcinoma detection: efficient use of computed tomography during arterial portography and during hepatic arteriography. J. Gastroenterol., 40, 266-273, 2005.
[18] Ueno, S., Aoki, D., Maeda, T., Kubo, F., Hokotate, H., Fukukura, Y., and Aikou, T.: Preoperative assessment of multicentric occurrence in synchronous small and multiple hepatocellular carcinoma based on image-patterns and histological grading of non-cancerous region. Hepatol. Res., 29, 24-30, 2004.

[19] Colagrande, S., Villa, G.L., Bartolucci, M., Lanini, F., Barletta, G., and Villari, N.: Spiral computed tomography versus ultrasound in the follow-up of cirrhotic patients previously treated for hepatocellular carcinoma: a prospective study. J. Hepatol., 39, 93-98, 2003. 